\title{
Regional prediction of landslide hazard using probability analysis of intense rainfall in the Hoa Binh province, Vietnam.
}

\begin{abstract}
The main objective of this study is to assess regional landslide hazards in the Hoa Binh province of Vietnam. A landslide inventory map was constructed from various sources with data mainly for a period of 21 years from 1990 to 2010. The historic inventory of these failures shows that rainfall is the main triggering factor in this region. The probability of the occurrence of episodes of rainfall and the rainfall threshold were deduced from records of rainfall for the aforementioned period. The rainfall threshold model was generated based on daily and cumulative values of antecedent rainfall of the landslide events. The result shows that 15-day antecedent rainfall gives the best fit for the existing landslides in the inventory. The rainfall threshold model was validated using the rainfall and landslide events that occurred in 2010 that were not considered in building the threshold model. The result was used for estimating temporal probability of a landslide to occur using a Poisson probability model. Prior to this work, five landslide susceptibility maps were constructed for the study area using support vector machines, logistic regression, evidential belief functions, Bayesianregularized neural networks, and neuro-fuzzy models. These susceptibility maps provide information on the spatial prediction probability of landslide occurrence in the area. Finally, landslide hazard maps were generated by integrating the spatial and the temporal probability of landslide. A total of 15 specific landslide hazard maps were generated considering three time periods of 1,3 , and 5 years.
\end{abstract}

Keyword: Landslide hazard; Rainfall threshold; GIS; Hoa Binh; Vietnam 